\title{
Automatic video fire detection approach based on PJF color modeling and spatio-temporal analysis
}

\author{
Zeineb Daoud \\ REsearch Groups in Intelligent \\ Machines \\ University of Sfax \\ National Engineering \\ School of Sfax \\ Sfax, 3038, Tunisia \\ zeineb.daoud.tn@ieee.org
}

\author{
Amal Ben Hamida \\ REsearch Groups in Intelligent \\ Machines \\ University of Sfax \\ National Engineering \\ School of Sfax \\ Sfax, 3038, Tunisia
}

benhamida.amal@gmail.com

\author{
Chokri Ben Amar \\ REsearch Groups in Intelligent \\ Machines \\ University of Sfax \\ National Engineering \\ School of Sfax \\ Sfax, 3038, Tunisia \\ chokri.benamar@ieee.org
}

\begin{abstract}
Recently, due to the huge damage caused by fires in many countries in the world, fire detection is getting more and more interest as an increasing important issue. Nowadays, the early fire detection in video surveillance scenes is emerging as an alternative solution to overcome the shortcomings of the current inefficient sensors. In this paper, we propose a new video based-fire detection method exploiting color and motion information of fire. Our approach consists in detecting all moving regions in the scene to select then areas likely to be fire. Further, motion analysis is required to identify the accurate fire regions. The proposed method is evaluated on different video datasets containing diverse fire and non-fire videos. Experimental results demonstrate the effectiveness of our proposed method by achieving high fire detection and low false alarms rates. Moreover, it greatly outperforms the related works with $98.81 \%$ accuracy and only $2 \%$ of false positive rate.
\end{abstract}

\section{Keywords}

Video fire detection, background subtraction, color modeling, PJF color space, spatio-temporal analysis.

\section{INTRODUCTION}

Natural and human-instigated disasters threaten on the one hand the environment, people's lives and livelihoods on the other. Fire has been one of the most destructive disasters leading to enormous suffering and hazardous effects. The 2018 summer saw an unusually high number of fires in many countries of the world such United States, Sweden, Portugal, etc [Min 18]. For instance, successively in 2017 and 2018, California was hit with the most destructive wildfires in its recorded history. According to the report presented in September 2018 by the California Department of Insurance, Carr Fire and Mendocino Complex are the largest ones [Eva18]. Carr Fire has destroyed 1077 homes, 22 commercial structures, and 500 outbuildings have been devastated with at least hundreds of people were killed. As reported in [Min 18], the Directorate General of Civil Security and Crisis Management (DGSCGC) of the French Ministry of the Interior announces that forest fire is covering large areas in Europe. For example, more

Permission to make digital or hard copies of all or part of this work for personal or classroom use is granted without fee provided that copies are not made or distributed for profit or commercial advantage and that copies bear this notice and the full citation on the first page. To copy otherwise, or republish, to post on servers or to redistribute to lists, requires prior specific permission and/or a fee. than 350000 ha in Portugal in 2017 compared to 24 500 ha in France. Therefore, there are significant social, economic and environmental impacts that the world is facing after fires, in spite the use of currently available fire sensors. These conventional sensors have been widely used for personal security and commercial applications. However, they usually take a long time to respond because carbon particles, smoke or heat require more time to reach the detector. Moreover, these traditional detectors are generally limited to indoor and are not applicable to outdoor in open large spaces such as forests [Çet13]. Most of them cannot provide additional information about fire location, dimension, etc [Çet13]. Face to the weaknesses of these sensors-based fire detection, fast and accurate fire detection methods are needed to protect both people and environment. Video Fire Detection (VFD) can be considered as a suitable solution especially with the propagation of video surveillance systems [ABe14, Ben16]. It has shown better flexibility, effectiveness and reliability [Han17], thanks to the capacity of image processing [Mej08, Gue11, Bou12] and of video processing techniques [Ela06, Kou12] to detect and analyze uncontrolled fire behavior in video surveillance scenes [ABe13, Ben13]. In fact, fire detection errors are reduced, and the response time can be immediate and faster than traditional sensors, as cameras do not need to wait for the smoke or heat diffusion. In addition, VFD systems are able to detect fire in large open scale 
areas providing crucial information to understand clearly the development of fire, such as size, growth and propagation [Çet13]. Consequently, VFD methods have received great attention by researchers in these recent years. They focus on developing VFD algorithms, based on exploiting fire characteristics to detect and analyze flames in consecutive video frames. In this context, our work is introduced. It aims to detect fire regions accurately by using color information and motion analysis. The main idea is a combination of background subtraction, to keep at the first stage only moving objects and a color model to identify then the possible fire regions based on PJF color space. Add to that, a motion descriptor, based on spatial and temporal analysis, is applied to filter out fire-colored objects. This paper is organized as follows: Section 2, presents the related works of fire detection. A description of the new fire detection approach is presented in section 3 . Section 4 provides experimental results and their discussions for system evaluation. Finally, conclusions are drawn in the last section.

\section{RELATED WORKS}

There are several methods in the literature developed for fire detection from video sequences. The current video-based fire detection systems use different fire features principally color, shape and motion. Based on these characteristics, the existing VFD systems can be classified into three categories: color-based, shapebased and motion-based.

Generally, almost all current methods employ color and motion information to detect the flame. The first works use purely color-based models. For instance, in [Che04], the RGB channels and the saturation values of the HSV color space are used. A set of rules is developed to determine fire pixels. These rules are based on the assumption that fire pixels have an intensity of red higher than green, and green higher than blue. However, this method does not well perform at distinguishing real fire regions from moving regions having similar fire color. To solve this problem, Çelik et al. in [Çel07] suggest to add the foreground object information to the statistical color model to detect fire pixels in RGB color space. This proposed fire detection method results in very high false alarms rates due to the brightness changes and its sensitivity to the tuning parameters employed in background subtraction. That is why, Çelik et al. propose in [Çel09] a chrominance model based on the $\mathrm{YC}_{\mathrm{b}} \mathrm{C}_{\mathrm{r}}$ color space, since it can distinguish bright regions more than other color spaces. The authors use the mean intensity of the pixels and the difference between the $\mathrm{C}_{\mathrm{b}}$ and $\mathrm{C}_{\mathrm{r}}$ channels to compose detection rules. Hence, their generic color model improves its robustness in segmenting fire regions. Motivated by the idea that $\mathrm{YC}_{\mathrm{b}} \mathrm{C}_{\mathrm{r}}$ is better in discriminating the luminance from chrominance, the fire recognition system suggested in [bin15], is based on a set of rules developed to identify the fire pixel values of $R, G, B$, $\mathrm{Y}, \mathrm{C}_{\mathrm{b}}$ and $\mathrm{C}_{\mathrm{r}}$ components in an image. Results show that combination between color spaces can better detect fire and confirm that $\mathrm{YC}_{\mathrm{b}} \mathrm{C}_{\mathrm{r}}$ color space is the best as to compare to RGB since it can separate luminance from chrominance more effectively than RGB. However, by applying this method, moving firecolored regions, in complicated environments such light reflections and brighter or darker environments are also detected. To deal with this issue, a different approach in [Zho15] consists in excluding color and investigating the shape features of flames and interference image. Eight candidate features are selected to establish weak classifier for each shape attributes. Results of this method show the inefficiency of applying just shape to detect fire regions. From these works, the majority of the onefeature based methods achieves seldom satisfactory detection results. It may be concluded that obviously using one feature by itself cannot be reliable to detect fire. In fact, many natural objects sharing the same characteristics with flame are wrongly detected. Besides, changes in the flame appearance and environmental conditions (weather conditions, visibility and time of day) in the scene complicate fire detection. Thereby, a combination of characteristics is needed to detect fire accurately with lower errors rate. Some works such [Yan12] combines color and shape information, based on the assumption that fire randomly changes its shape during its propagation, to be able to distinguish fire from non-fire objects. In the same way, Xi Zhang et al. merge in [XiZ12] color and shape features. Fourier descriptors and edge corrosion model are used to recognize fire regions after its extraction by a mixture of Gaussian distributions in HSV color space. Another approach presented in [Cho10] adds fire's texture to color and shape features. Assuming that fire regions have a significant amount of texture characteristics because of its random nature, the proposed method is useful to differentiate between fire and non-fire regions such as autumn color leaves. Although adding shape or texture information to color improves fire detection, many fire colored objects still detected. It can be observed that using color only or combined with shape is not enough owing to the presence of moving objects having the same color or shape features of fire such as red cars and persons with red clothes. To face this issue, the distinctive disordered movement of fire regions must be taken into account. It is a pertinent way of differentiating moving rigid objects from red colored regions to enhance the detection rate. As example in [Han 17], a new method for detecting fire based on motion feature and color information is proposed. Motion detection using Gaussian Mixture Model (GMM) for background subtraction is applied to extract moving objects from the scene. Then, multi-color-based 
detection combining the RGB, HIS and YUV color spaces is employed to filter out non-fire regions. Once fire regions are extracted, foreground objects with similar fire colors or caused by color shifting are also selected. To remove these spurious fire-like regions, Kim Heegwang et al. propose in [Kim16] a different approach using color and motion estimation. It consists in extracting candidate regions in the HSI color space to estimate them using optical flow. Then, the motion vectors are quantized into eight directions and fire regions are detected when the amount of the quantized motion vectors exceeds a fixed threshold. The proposed system outperforms the color-based and the shape-based methods in terms of detection performance. As flame detection remains a challenging issue due to the fact that many natural objects have similar characteristics with fire, a new algorithm for video-based flame detection, which employs various spatio-temporal features such as color probability, contour irregularity, spatial energy, flickering and spatio-temporal energy is presented in [Dim12] by Dimitropoulos et al. They suggest adding in [Bar13] a new spatio-temporal consistency to combine it with the features extracted in [Dim12]. Texture feature in [Dim15] is also added to their fire detection system to increase its robustness. Thus, video-based fire detection systems using multifeatures of fire are more efficient than systems based on only one feature. Moreover, in [Fog15a], a multiexpert system is developed by combining three descriptors based on color, shape and motion. A set of rules are implemented in the YUV color space to distinguish fire colored objects. Moving regions are detected as blobs via the scale-invariant feature transform (SIFT) descriptor. And, shape variation of the minimum bounding boxes which enclose the detected blobs is also used. Similarly, Li et al. design in [LiS17] a multi-attribute-based fire detection system which combines the color, geometric, and motion attributes. Two novel descriptors to characterize the geometric and motion features of fire are developed, the first one represents the outline of fire using contour moment and line detection and the other captures the instantaneous motion of fire with dense optical flow to well represent the inside and the boundary motions of fire. Despite of the capacity of these methods to detect fire regions, they still have limited application and lack enough robustness. They cannot effectively detect fire because of i) many natural objects sharing the same characteristics with the flame, ii) large variations of flame appearance in videos and iii) environmental changes that complicate fire detection, for example, shadows, clouds, sun shining, car lighting, light reflections or other kinds of lights can behave or flicker like fire [Bar13]. In order to overcome these limitations, a VFD method, which employs color information and motion analysis, is proposed in this paper.

\section{THE NEW FIRE DETECTION APPROACH}

By reviewing the above works, we aim to develop a video-based fire detection approach to reach high detection rate and low false alarms rate. The VFD method suggested in this work is basically composed of three phases as illustrated in figure 1 .

$\underset{\text { Video }}{\text { Input }} \begin{gathered}\text { Moving } \\ \text { Regions } \\ \text { Detection }\end{gathered} \rightarrow \sqrt[\begin{array}{l}\text { Candidate } \\ \text { Regions } \\ \text { Selection }\end{array}]{\text { Selion }}$

Figure 1. Flow chart of the presented fire detection method.

Moving regions are firstly detected by using a background subtraction algorithm. Then, a novel model in PJF color space is developed to select fire candidate regions. Spatio-temporal analysis is finally applied in order to distinguish fire regions from other fire-colored objects.

\subsection{Moving regions detection}

Detection of moving regions is a necessary step in VFD. Since fire can be naturally interpreted as a moving object in the video, several methods such as the Gaussian Mixture Model (GMM) in [LiS17] [Han17] and frame differences in [Pel18] [See14] are used to detect moving regions. Nevertheless, the most popular VFD systems are based on background subtraction methods to identify moving objects by separating foreground from background in video sequences. Thanks to their simplicity, real time performing and low computational cost, the Gaussian Mixture Models are widely used to create background models and estimate moving objects. We adopt in our approach the Global Minimum with a Guarantee (GMG) algorithm from the GMM family [God12]. It employs probabilistic foreground segmentation algorithm that identifies possible foreground objects to track them later on. In fact, GMG combines statistical background image estimation, per-pixel Bayesian segmentation, morphological filtering operations like closing and opening to remove inherent noise, and an approximate solution to the multi-target tracking problem using a bank of Kalman filters [Kal60] and Gale-Shapley matching [Gal62]. A heuristic confidence model enables selective filtering of tracks based on dynamic data. Figure 2, presented below, illustrates its system block diagram.

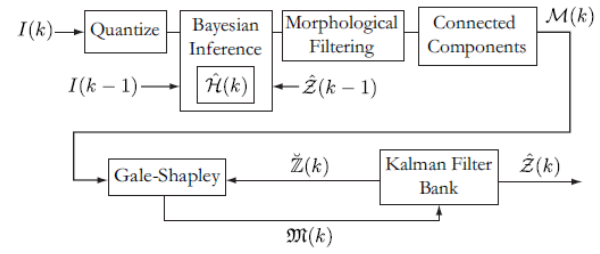

Figure 2. GMG algorithm block diagram [God12].

The GMG algorithm proves its robustness during variable lighting conditions and sudden changes in the 
appearance of the background. From the detected moving regions after the GMG application, we aim to select only entities having the same fire's color characteristics. That's why we suggest using the color features of the moving areas to discriminate between fire and non-fire regions.

\subsection{Candidate regions selection}

Selection of candidate regions is the second stage in our approach for fire detection with the aim of distinguishing the fire regions from the other moving objects. It is based on the most distinctive characteristic of fire, which is the color. Therefore, we propose a new color-based model in the PJF color space [Jac12].

\subsubsection{PJF color space}

The idea behind the PJF color space was transforming the RGB values into a new color space that follows more closely the concept of $\mathrm{L}^{*} \mathrm{a}^{*} \mathrm{~b}^{*}$ by expressing brightness as a single variable. This color space expresses color in firstly a variable that runs from green to red and secondly with a variable from blue to yellow, while remaining vectors are inside the RGB color cube as depicted in figure 4 [Jac12]. That's why PJF reflects better the structure of $\mathrm{L}^{*} \mathrm{a} * \mathrm{~b}^{*}$ color space by achieving a low color calibration error.

Given RGB data, the conversion to PJF color space is formulated as follows [Jac12]:

$$
\begin{gathered}
P=\sqrt{R^{2}+G^{2}+B^{2}} \\
J=R-G \\
F=R+G-B
\end{gathered}
$$

Where the component $\mathrm{P}$ measures the magnitude of brightness, the second component $\mathrm{J}$ indicates the relative amounts of red and green and the third one $\mathrm{F}$ measures simply the relative amounts of yellow and blue. The new color channels produced by equations (1), (2) and (3) are shown in figure 3 and the PJF color space model is presented in figure 4 .

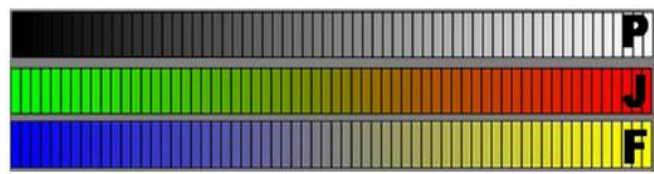

Figure 3. The three-color channels created by the color space transform [Jac12].

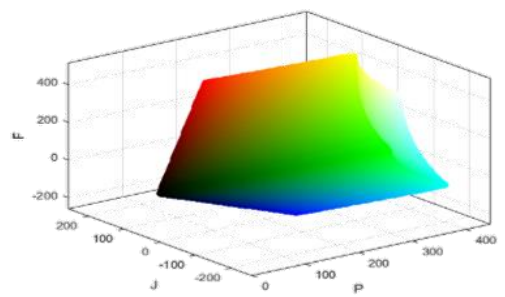

Figure 4. 3D distribution of the PJF color space.

It is assumed that fire's color is generally close to the red color with higher illumination values. Through
PJF color space, the representation of fire color information is better than other color spaces since margin's variation of the fire color is presented by its two components J and F [Dus15]. Based on these cited statements, we propose to define rules in PJF color space to select fire regions.

\subsubsection{Fire color-based model}

A specific color spectrum characterizes fire where its natural colors are often in the red-yellow range. As mentioned above, the $\mathrm{J}$ channel in the PJF color space is the difference between red and green, and indicates the relative amounts of these two colors. Likewise, the F channel measures the relative amounts of yellow and blue. For these reasons fire pixels belong clearly in the J-F plane as confirmed in Fig. 4. Hence, these two PJF color space components can be used to estimate the range of flame pixels. In RGB image, fire is a red colored light source. Therefore, the red value $R$ for each pixel in fire region must be over a threshold as presented in equation (4) [Che04].

$$
R>R_{T}
$$

Where $R_{T}$ denotes the threshold value for the red channel.

Motivated by these facts and based on the two transformation equations (2) and (3), the proposed rule to select the candidate regions of fire from other moving objects is given below:

$$
F \geq 2 R_{T}-J
$$

Where $R_{T}$ is experimentally fixed to 120 [Che04]. At this stage, fire detection leads to high false alarms. The selected candidate regions of fire may still be non-fire because of several moving objects having the same colors as fire such persons wearing red clothes or carrying red artifacts, red vehicles, etc. Consequently, analysis of fire motion are needed to filter the obtained regions.

\subsection{Spatio-temporal analysis}

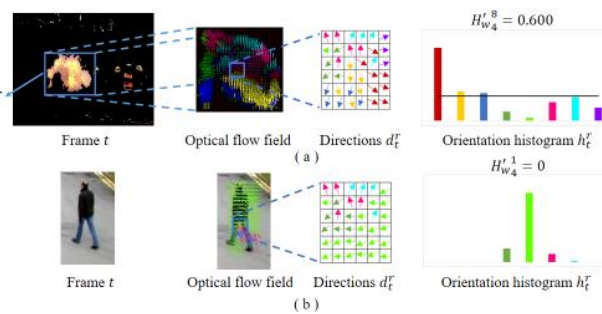

\section{Figure 5. Optical flow fields and orientation histograms for (a) fire region and for (b) pedestrian object.}

Motion is an essential characteristic that should be merged with color to detect accurately fire regions. It is clearly that the fire's motion generally differs from the motion of the irrelevant entities since it is characterized by its disordered movement. Thus, motion analysis is needed to effectively discriminate 
these areas. It consists mainly of three steps described as follows: (1) computation of motion vectors, (2) spatial analysis and (3) temporal analysis to finally detect fire regions.

\subsubsection{Computation of motion vectors}

To analyze the motion of the selected regions, we firstly compute the optical flow field. Motion information is obtained by calculating the velocity vectors for each pixel $(x, y)$ in region $r$ at frame $t$. These motion vectors are got by using Farneback method [Far03]. A two-frame motion estimation algorithm uses firstly polynomial expansion, where a neighborhood of each image pixel is approximated by quadratic polynomials. Afterwards, considering these quadratic polynomials, a new signal is constructed by a global displacement that is calculated by equating the coefficients in the quadratic polynomials' yields. The optical flow field is described by $\left(V x_{t}^{r}(x, y), V y_{t}^{r}(x, y)\right) \quad$ where $V x_{t}^{r}(x, y)$ and $V y_{t}^{r}(x, y)$ denote the horizontal and vertical velocities respectively of the region $r$ at frame $t$.

\subsubsection{Spatial analysis}

By adopting the fact that fire movements are multidirectional, we aim to find fire areas. The motion directions of each pixel of the selected candidate regions are firstly extracted from every frame, by using optical flow field obtained at the previous step. The direction $d_{t}^{r}$ of each pixel $(x, y)$ is then computed via the following equation:

$$
d_{t}^{r}(x, y)=\tan ^{-1}\left(\frac{V y_{t}^{r}(x, y)}{V x_{t}^{r}(x, y)}\right)
$$

Where $r$ is the selected candidate region in frame $t$. For a better characterization of the acquired directions, they are quantized to $N_{d}$ levels as shown in figure 6 where $N_{d}$ is set to 8 .

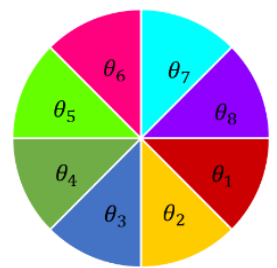

Figure 6. Quantization of moving directions.

In order to discriminate fire and non-fire regions, the different directions are interpreted by computing the orientation histogram $h_{t}^{r}$ for each candidate region $r$ in frame $t$. The appearance of histograms as represented in the example of Fig. 5(a) confirms that the movement of the fire is disordered and spreads around the fire region, hence the dispersion of its orientation histogram. In contrast, the motion of nonfire objects, like the pedestrian in Fig. 5(b), is distributed in uniform directions, which explains that its orientation histogram is concentrated in few bins. Through the orientation histograms interpretation, we can conclude that a dispersed histogram generated with more bins corresponds to fire region. However, using orientation histograms may be insufficient to detect fire regions because of the similar histogram's appearance of some non-fire objects like autumn color leaves, which have disordered movement. That's why we propose to compute the normalized weighted entropy $H_{w_{t}}^{\prime r}$ which is obtained by calculating first of all the weighted entropy $H_{w_{t}}^{r}$ defined as follows:

$$
H_{w_{t}}^{r}=-W_{t}^{r} * \sum_{\theta_{j}=1}^{N_{d}} p_{t}^{r}\left(\theta_{j}\right) \cdot \log _{2}\left(p_{t}^{r}\left(\theta_{j}\right)\right)
$$

Where $r$ is the region of interest at frame $t, p_{t}^{r}$ represents the orientation probability of each quantified direction $\theta_{j}, j=1, \ldots, N_{d}$, of the motion vector. $W_{t}^{r}$ denotes the weight which aims to maintain a deviation between the entropy of fire regions and that of non-fire in the interest of distinguishing easily between areas. That means, $W_{t}^{r}$ enhances the entropy of fire region and reduces the entropy of non-fire region. It is calculated using the orientation histogram by counting the number of the most frequent bins with a greater amount of information as shown in the following equation:

$$
\begin{gathered}
W_{t}^{r}=\operatorname{Card}\left\{h_{t}^{r}\left(\theta_{j}\right), \mathrm{j}=1 \ldots N_{d} \text { with } h_{t}^{r}\left(\theta_{j}\right)>\right. \\
\left.\frac{\sum_{\theta_{j}=1}^{N_{d}} h_{t}^{r}\left(\theta_{j}\right)}{N_{d}}\right\}
\end{gathered}
$$

We normalize then the range of values of $H_{w_{t}}^{r}$ by using min-max normalization to scale them in $[0,1]$ to obtain $H_{w_{t}}^{\prime}$.

\subsubsection{Temporal analysis}

Fire region in certain single frame may not be detected because of the flickering effects and instability of the motion. Flickering is one of the main features of flame and it is very significant to discriminate between fire regions and fire-colored objects. Besides, the motion of non-fire objects, like human and vehicles, is regular, hence it is temporally continuous and uniform. Differently to other entities, fire is always in disordered and discontinuous motion due to the flickering effects. For these reasons, temporal analysis is applied to each candidate region. After calculating normalized weighted entropy $H_{w_{t}}^{\prime r}$ in the previous step, the candidate areas must be further verified using movement's temporal variation since motion analysis in a single frame are not enough to identify fire. For each candidate region $r$, we suggest to calculate, in equation (9), the temporal entropy $H_{T}(r)$ over the consecutive $N$ frames as follows:

$$
H_{T}(\mathrm{r})=\frac{\sum_{t=1}^{N} H_{w_{t}}^{\prime r}(r)}{N}
$$

Where $H_{w_{t}}^{\prime r}$ is the normalized weighted entropy of region $r$ at frame $t$ and $N$ represents the number of frames. At this point, a threshold $\tau$ will be determined to distinguish fire regions. As shown in equation (10), 
if $H_{T}(\mathrm{r})$ is greater than a given $\tau$, the region $r$ is classified as fire.

$$
r=\left\{\begin{array}{c}
\text { fire if } H_{T}(r) \geq \tau \\
\text { non fire if } H_{T}(r)<\tau
\end{array}\right.
$$

\section{EXPERIMENTAL RESULTS}

In this section, we evaluate our approach on a wide dataset. Moreover, we carry out comparison with two recent fire detection methods [LiS17] [Pel18].

\subsection{Dataset}

In spite of the importance of fire detection researches nowadays, there are no standard benchmark datasets for fire detection up to now. Therefore, to test the presented approach we construct our database from four public datasets available in [Fog 15b] [Çet07] [Caz17] [Kmu18]. It contains 157 videos varied between 10 and $30 \mathrm{fps}$, in a diverse range of environmental conditions and illumination: 137 positive videos characterized by the presence of fire and 20 negative videos, which do not contain fires. Hence, 246581 frames compose this collection.

\subsection{Evaluation metrics}

In order to measure the robustness of our new method, it is necessary to compute validation metrics. True positive $(T P)$, false positive $(F P)$, true negative $(T N)$ and false negative $(F N)$ are calculated. $T P$ is the number of frames where fire regions are correctly detected. FP is the number of frames in which non-fire regions are incorrectly detected as fire. $T N$ is the number of frames with non-fire regions that are correctly rejected and $F N$ is the number of frames in which fire regions are wrongly rejected. For negative fire videos, the evaluation is done by using the detection error rate $E R$ calculated in the equation (11) presented below. It represents the rate of recognizing fire in a frame where there is no fire. TNR and FPR metrics are also used to measure respectively the ability of the algorithm to well eliminate non-fire regions and the false alarms rate.

$$
E R=\frac{F P}{\text { Total Number of Frames }}
$$

Similarly, positive fire videos are assessed with different metrics to measure the effectiveness of detecting fire regions by the proposed method. As a first metric, we use the detection rate $D R$; it is defined as the rate of detecting fire successfully by the following equation:

$$
D R=\frac{T P}{\text { Total Number of Frames }}
$$

Accuracy is also employed to measure how close our algorithm can detect the correct fire areas. It is calculated via the equation (13) below:

$$
\text { Accuracy }=\frac{T P+T N}{T P+T N+F P+F N}
$$

Recall and precision are calculated too, as stated in equations (14) and (15). Recall metric, named also sensitivity, expresses the ability of an algorithm to find all the pertinent cases. While, precision measures the proportion of detections that a method indicates as relevant when they are really relevant.

$$
\begin{gathered}
\text { Recall }=\frac{T P}{T P+F N} \\
\text { Precision }=\frac{T P}{T P+F P}
\end{gathered}
$$

In order to measure the trade-off between precision and recall, we combine the two metrics using the $F_{1}$ score in the following equation. It is a weighted harmonic mean showing how many frames with fire regions are correctly detected.

$$
F_{1} \text { score }=2 * \frac{\text { Recall } * \text { Precision }}{\text { Recall }+ \text { Precision }}
$$

\subsection{Evaluation of the new fire detection approach}

It should be noted that the number of frames $N$ used to calculate the temporal entropy for each candidate area (cited in the section 3.3.3), is experimentally set to 20 according to results presented in Fig. 7. This figure shows the variation of the fire detection accuracy of different fire videos samples to validate the choice of $N$ frames. For example, the first scene (Scene_1) achieves in terms of accuracy $0.84,0.88,0.83$, and 0.76 with $10,20,30,40$ and 50 frames respectively. It is discernable from Fig. 7 that increasing $N$ decreases the accuracy values. The best rates are obtained when $N$ is equal to 20 . As a consequence, we adopt $N=20$ to evaluate our method.

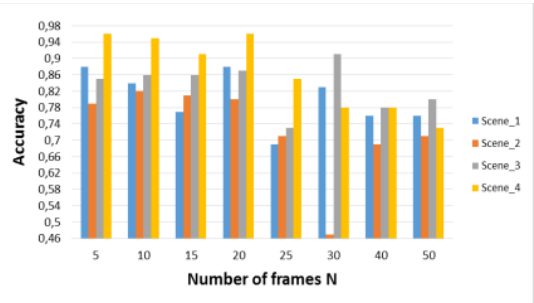

Figure 7. Comparison of fire detection accuracy in video sequences by varying the number of frames $N$.

In this section, we aim at successfully detecting fire regions in several scenes to measure the performance of our video-based fire detection approach. Figure 9 illustrates the output frames of each phase of our approach. On the one hand, Fig. 9(A) shows the results on three different positive fire movies. It can be remarkable that our presented method is able to eliminate non-fire moving objects, such as in video A2. These include fire-like colored objects, such the firefighters wearing jackets and caps with similar color of fire. In the same way, fire-color background areas, which may cause false detection like scene A1 
in Fig. 9(A), are rejected too. Moreover, fires in videos, which are filmed in different conditions of environment, weather and illumination (in forest, indoor, outdoor, day and night scenes), are successfully detected. On the other hand, results on negative videos where there is no fire are presented in Fig. 9(B). Spurious fire-like objects such as sun reflections, human with red clothes and bright sparkling lights are correctly rejected. Scene B3 of Fig. 9(B) proves that spatial analysis at Fig. 9(B3.c) (as detailed in the section 3.3.2) are not sufficient to distinguish fire regions from areas sharing the same fire's characteristics. As it is shown in Fig. 9(B3.b) and Fig. 9(B3.c) bright sparkling lights are still detected after applying our color model. But, adding temporal analysis improves differentiation between regions. As depicted in Fig. 9(B3.d), all fire colored areas are rejected. This further confirms the robustness of our approach in recognizing fire regions.

From these results, we can conclude that our proposed method succeeds in distinguishing fire from non-fire regions. This is thanks to the fact that the deviation between the temporal entropy $H_{T}$ values of fire and that of non-fire regions has become more larger. In fact, they can be easily differentiated. These results are clearly observed in figure 8 , which shows the variation of temporal entropy for fire and non-fire areas.

As it is illustrated, values of temporal entropy for fire region (the red curve) is greater than those of non-fire. Through the analysis of the variation of temporal entropy in figure 8 , discriminating between moving regions is becoming easier. It is sufficient to determine the threshold $\tau$ (cited in the section 3.3.3). Otherwise, $H_{T}$ of fire area is over than $\tau$. This evidently confirms that fire is always in active motion and its temporal entropy is near to 1. As it is shown in Fig. 5(a), a fire region has a temporal entropy value equal to 0.832 .

(A) Positive scenes
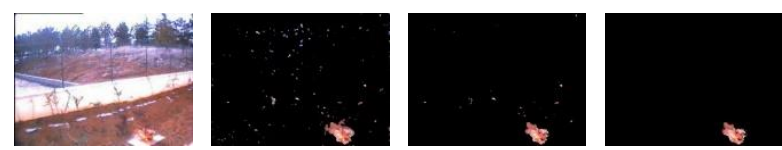

A1. a
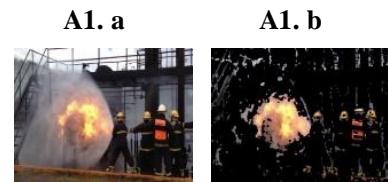

A1. c

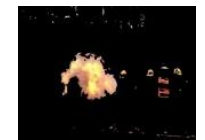

A1. d

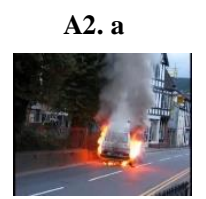

A2. b

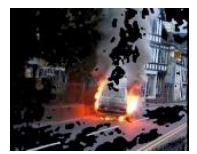

A3. b

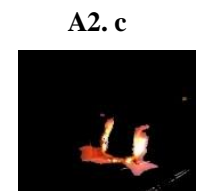

A3. c

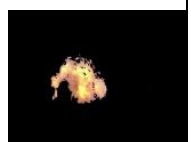

A2. $d$

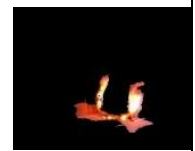

A3. d

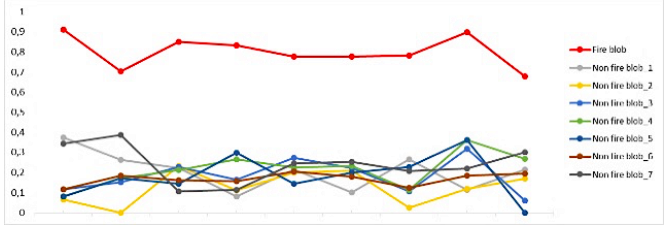

Figure 8. Temporal variation of entropy for fire region and non-fire areas.

However, for areas which may be a moving objects having the same colors as fire, their temporal entropy is close to 0 . For example, $H_{T}$ value for a non-fire area is equal to 0.165 . As earlier detailed, these values demonstrate effectively that the movement of firecolored regions is uniform and significantly different from the motion of fire regions. That's why fire areas have a higher value of temporal entropy $H_{T}$. In this study, our experiments show that the best discrimination is achieved when the value of the used threshold $\tau$ is fixed to 0.5 .

The assessment of this approach is given below, and results are shown in Table 1 and Table 2. For almost positive fire movies, our novel method has yielded a true positive $(T P)$ average of $99 \%$ for detecting the fire regions correctly, hence the detection rate $D R$ is high. Values of false positive $(F P)$ are null which leads to a null detection error rate $E R$, this proves how effectively our algorithm can distinguish fire from non-fire regions. Precision and recall values in Table 1 reach over 0.9. Indeed, the obtained values of recall are respectable, they are around of 0.99. High precision relates to the low false positive rate. This proves also that almost all fire regions are detected correctly. Most significantly, the overall performance of the presented approach is high in terms of the weighted harmonic mean $F_{1}$ score with 0.99 . Add to that, Table 2 shows the achieved results on the accuracy of fire detection in three negative fire scenes.

(B) Negative scenes
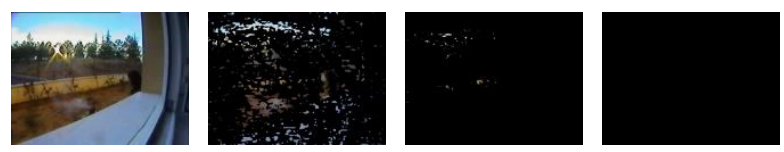

B1. a

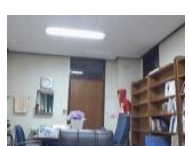

B1. b

B1. c
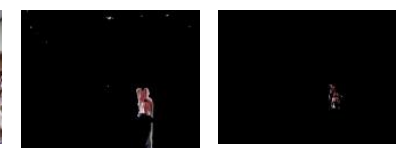

B1. d
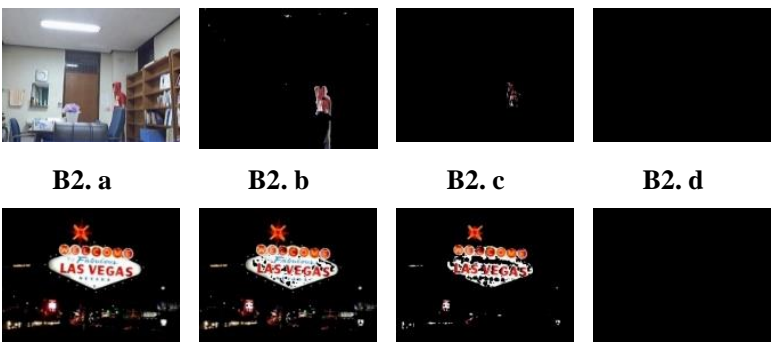

B2. b

B2. c

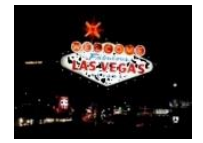

B3. b

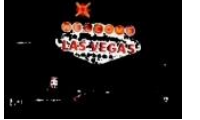

B3. c

Figure 9. Results of the presented fire detection method on (A) positive fire videos and on (B) negative

movies. (a) Original frames, (b) Moving regions detection using the GMG algorithm, (c) Candidate regions selection by applying the PJF color model, (d) Detected fire regions obtained after spatio-temporal analysis. 


\begin{tabular}{|c|c|c|c|c|c|c|c|c|c|}
\hline Videos & ТP (\%) & $\mathrm{FP}(\%)$ & $\mathrm{DR}(\%)$ & $\mathrm{ER}(\%)$ & Recall & Accuracy & Precision & $\mathrm{F}_{1}$ score & $\begin{array}{l}\text { Sample } \\
\text { picture }\end{array}$ \\
\hline fireVid_001 & 98 & 0 & 98 & 0 & 0.98 & 0.98 & 1 & 0.98 & \\
\hline fire_8 & 99 & 0 & 99 & 0 & 0.99 & 0.99 & 1 & 0.99 & \\
\hline flame_1 & 100 & 0 & 100 & 0 & 1 & 1 & 1 & 1 & \\
\hline fire_11 & 99 & 0 & 99 & 0 & 0.99 & 0.99 & 1 & 0.99 & \\
\hline rescuer_060 & 96 & 0 & 96 & 0 & 0.96 & 0.96 & 1 & 0.98 & \\
\hline rescuer_021 & 100 & 0 & 100 & 0 & 1 & 1 & 1 & 1 & \\
\hline Average & 98.66 & 0 & 98.66 & 0 & 0.99 & 0.99 & 1 & 0.99 & \\
\hline
\end{tabular}

Table 1. Fire detection results of the presented method applied on positive fire movies

\begin{tabular}{|c|c|c|c|c|c|c|c|}
\hline Videos & TN (\%) & FN (\%) & ER (\%) & TNR & FPR & Accuracy & $\begin{array}{c}\text { Sample } \\
\text { picture }\end{array}$ \\
\hline fire_15 & 100 & 0 & 0 & 1 & 0 & 1 & \\
\hline fire_16 & 100 & 0 & 0 & 1 & 0 & 1 & \\
\hline fireworks & 94 & 0 & 6 & 1 & 0.06 & 0.94 & \\
\hline Average & 98 & 0 & 2 & 1 & 0.02 & 0.98 & \\
\hline
\end{tabular}

Table 2. False fire detection results of the presented method applied on negative fire movies

Since there is no fire, $F N$ of the proposed algorithm is null. $T N$ average of $98 \%$ is obtained resulting firstly in a greater $T N R$, secondly in a higher accuracy with 0.98 and lastly in a lower error rate with $2 \%$. These values reflect the interesting performance of our contribution that is to say it can distinguish accurately fire regions from non-fire areas having the same colors as fire.

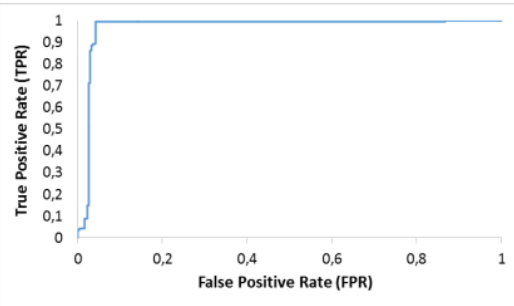

Figure 10. Receiver Operation Characteristics Curve.

As well, the performance is depicted in figure 10 where the Receiver Operating Characteristics (ROC) curve is presented. This curve shows how much the novel approach is able to discriminate between fire and non-fire regions. The area under the ROC curve is a pertinent indicator for performance measuring. The higher is the Area Under the Curve (AUC) the better is the algorithm at distinguishing between fire regions and fire-like colored objects [Faw06]. For this, the AUC computed value is 0.969 . Therefore, the ROC curve proves too that our method has a low false alarms rate. From these results, we can conclude that the proposed approach accomplishes a rate of accuracy values over than 0.9 for both positive and negative scenes of the datasets, and only $2 \%$ for the false alarms rate.

\begin{tabular}{|c|c|c|c|}
\hline Method & Accuracy & FPR & FNR \\
\hline$[$ Pel18] & $64.30 \%$ & - & - \\
\hline$[$ LiS17] & $92.30 \%$ & $8.33 \%$ & $9.09 \%$ \\
\hline Presented & $98.81 \%$ & $2 \%$ & $1 \%$ \\
\hline
\end{tabular}

Table 3. Comparison of the presented approach with two related works

For better assessment, we compare the presented approach with other fire detection methods based on motion and color features. In [LiS17], a multiattribute-based fire detection system is developed which combines the color, geometric, and motion attributes. While, [Pel18] is based on color segmentation and moving objects detection by applying the frame differences. The experimental results are illustrated in Table 3.

It should be noted that accuracy, FPR and FNR values presented in Table 3 are the averages of all used datasets. FPR and FNR values obtained with our contribution are better than [LiS17] method with a decrease from $8.33 \%$ to $2 \%$ and from $9.09 \%$ to $1 \%$ respectively. These values are very low that is to say that our system can distinguish between fire regions and fire-like colored objects in the same frame, detect very well fire regions and eliminate undesirable areas. From Table 3, it can be found that our results significantly outperform Li's [LiS17] and Peleshko's 
methods [Pel18] in accuracy rate with the highest value of $98.81 \%$. By combining the PJF color model, the motion information and the spatio-temporal analysis of the fire's behavior, the obtained accuracy is nearly $6.5 \%$ and $34.5 \%$ greater than the related works [LiS17] and [Pel18] respectively. As shown in the first row of Table 3, accuracy in [Pel18] is 64.30\%, because of using only color segmentation with frame differences. It cannot correctly discriminate fire from non-fire moving objects. Whereas, although Li's method in [LiS17] accomplishes $92.30 \%$ of accuracy, it may yield a few failure cases on some sequences (long distance between fire and the camera). In these cases, the geometric and motion attributes cannot work well because the fire areas are too small. For our method, it is able to detect fire regions in these special cases despite the far distance. Compared to the motion attribute in [LiS17], motion analysis in our work can obviously improve the detection accuracy by using spatial and temporal information. Generally, the proposed approach achieves a promising performance in detecting fire.

\section{CONCLUSION}

In this paper, we present an effective novel approach for fire detection in video sequences. It is based on color and motion features. It consists in detecting moving regions at the first stage using the GMG algorithm for background subtraction. After that, a novel fire color model developed in PJF color space selects candidate regions. Therefore, the detected fire regions are determined by a spatio-temporal analysis of the fire's motion. This method is tested over different datasets. It is shown experimentally that we succeed in detecting fire regions with accurate discrimination between fire and non-fire areas. Through experiments, we demonstrate that the achieved false alarms rate is only $2 \%$. The false negative rate where fire regions are wrongly rejected is reduced too to reach $1 \%$. Experimental results show also that our new method clearly outperforms the related works in terms of fire detection accuracy. In order to face the variable illumination problem, our approach can be further improved in the future. And the forthcoming scope includes the application of deep-learning methods.

\section{ACKNOWLEDGMENTS}

The research leading to these results has received funding from the Ministry of Higher Education and Scientific Research of Tunisia under the grant agreement number LR11ES48.

\section{REFERENCES}

[ABe13] A. Ben Hamida, M. Koubaa, H. Nicolas and C. Ben Amar, "Video pre-analyzing and coding in the context of video surveillance applications," 2013 IEEE International Conference on Multimedia and Expo Workshops (ICMEW), San Jose, CA, pp. 1-4, 2013.
[ABe14] A. Ben Hamida, M. Koubaa, C. Ben Amar and H. Nicolas, "Toward scalable application-oriented video surveillance systems," Science and Information Conference, London, pp. 384-388, 2014.

[Bar13] Barmpoutis, P., Dimitropoulos, K. \& Grammalidis, $\mathrm{N}$. Real time video fire detection using spatio-temporal consistency energy. in 2013 10th IEEE International Conference on Advanced Video and Signal Based Surveillance 365-370, IEEE, 2013.

[Ben13] Ben Hamida A, Koubaa M, Nicolas H, Ben Amar C. Spatio-temporal video filtering for video surveillance applications. In: IEEE International Conference on Multimedia and Expo Workshops (ICMEW),pp 1-6, 2013.

[Ben16] Ben Hamida, A., Koubaa, M., Nicolas, H., Ben Amar, C. Video surveillance system based on a scalable application-oriented architecture. Multimedia Tools and Applications, 75 (24), pp. 17187-17213, 2016.

[bin15] binti Zaidi, N. I., binti Lokman, N. A. A., bin Daud, M. R., Achmad, H. \& Chia, K. A. Fire recognition using RGB and YCbCr color space. ARPN J. Eng. Appl. Sci. 10, 9786-9790, 2015.

[Bou12] Boughrara, H., Chtourou, M., Amar, C.B. MLP neural network based face recognition system using constructive training algorithm. Proceedings of 2012 International Conference on Multimedia Computing and Systems, ICMCS 2012, pp. 233-238, 2012.

[Caz17] Cazzolato, M. T., Avalhais, L. P., Chino, D. Y., Ramos, J. S., de Souza, J. A., Rodrigues-Jr, J. F., \& Traina, A. J. FiSmo: A Compilation of Datasets from Emergency Situations for Fire and Smoke Analysis. Proc. Satell. events, 2017. Computer vision based fire detection database. [Online]. Available: https://goo.gl/uW7LxW. Accessed August 2018.

[Çel07] Çelik, T., Demirel, H., Ozkaramanli, H. \& Uyguroglu, M. Fire detection using statistical color model in video sequences. J. Vis. Commun. Image Represent. 18, 176-185, 2007.

[Çel09] Çelik, T. \& Demirel, H. Fire detection in video sequences using a generic color model. Fire Saf. J. 44, 147-158, 2009.

[Çet07] Çetin, A. E. Computer vision based fire detection software, 2007. Database. [Online]. Available: http://signal.ee.bilkent.edu.tr/VisiFire/. Accessed January 2018.

[Çet13] Çetin, A. E., Dimitropoulos, K., Gouverneur, B., Grammalidis, N., Günay, O., Habiboğlu, Y. H., \& Verstockt, S. Video fire detection-review. Digit. Signal Process. 23, 1827-1843, 2013.

[Che04] Chen, T.-H., Wu, P.-H. \& Chiou, Y.-C. An early fire-detection method based on image processing. in 2004 International Conference on Image Processing, 2004. ICIP'04. 3, 1707-1710, IEEE, 2004.

[Cho10] Choi, H.-W., Min, I.-K., Oh, E.-S. \& Park, D.-H. A study on the algorithm for fire recognition for automatic forest fire detection: The international conference on control, automation and systems 2010 (iccas 2010). in ICCAS 2010 2086-2089, IEEE, 2010.

[Dim12] Dimitropoulos, K., Tsalakanidou, F. \& 
Grammalidis, N. Flame Detection for Video-based Early Fire Warning Systems and 3D Visualization of Fire Propagation. in 13th IASTED International Conference on Computer Graphics and Imaging (CGIM 2012), Crete, Greece, 2012.

[Dim15] Dimitropoulos, K., Barmpoutis, P. \& Grammalidis, N. Spatio-temporal flame modeling and dynamic texture analysis for automatic video-based fire detection. IEEE Trans. Circuits Syst. Video Technol. 25, 339-351, 2015.

[DuS15] Du S.-Y.a \& c, L. Z.-G. . A comparative study of different color spaces in computer-vision-based flame detection. Multimed. Tools Appl. 75, 10291-10310, 2015.

[Ela06] El'Arbi, M., Ben Amar, C., Nicolas, H. Video watermarking based on neural networks. 2006 IEEE International Conference on Multimedia and Expo, ICME 2006 - Proceedings, pp. 1577-1580, 2006.

[Eva18] Evan Mills, Ted Lamm, Sadaf Sukhia, Ethan Elkind, and Aaron Ezroj. Trial by Fire: Managing Climate Risks Facing Insurers in the Golden State. Sacramento, CA: California Department of Insurance, 2018. Available at: https://www.law.berkeley.edu/wpcontent/uploads/2018/09/Trial-by-Fire-September2018.pdf.

[Far03] Farnebäck, G. Two-frame motion estimation based on polynomial expansion. in Scandinavian conference on Image analysis 363-370, Springer, 2003.

[Faw06] Fawcett, T. An introduction to ROC analysis. Pattern Recognit. Lett. 27, 861-874, 2006.

[Fog15a] Foggia, P., Saggese, A. \& Vento, M. Real-Time Fire Detection for Video-Surveillance Applications Using a Combination of Experts Based on Color , Shape, and Motion. IEEE Trans. Circuits Syst. Video Technol. 25, 1545-1556, 2015.

[Fog15b] Foggia, P. Computer vision based fire detection dataset. [Online]. Available: http://mivia.unisa.it/datasets/video-analysisdatasets/fire-detection-dataset/. Accessed December 2017.

[Gal62] Gale, D and Shapley, L. D. College admissions and the stability of marriage. Am. Math. Mon. 69, 9-15, 1962.

[God12] Godbehere, A. B., Matsukawa, A. \& Goldberg, K. Visual tracking of human visitors under variablelighting conditions for a responsive audio art installation. in 2012 American Control Conference (ACC) 4305-4312, IEEE, 2012.

[Gue11] Guedri, B., Zaied, M., Ben Amar, C. Indexing and images retrieval by content. Proceedings of the 2011 International Conference on High Performance Computing and Simulation, HPCS 2011, pp. 369-375, 2011.

[Han17] Han, X.-F. et al. Video fire detection based on gaussian mixture model and multi-color features. Signal, Image Video Process. 11, 1419-1425, 2017.

[Jac12] Jackman, P., Sun, D.-W. \& ElMasry, G. Robust colour calibration of an imaging system using a colour space transform and advanced regression modelling. Meat Sci. 91, 402-407, 2012.
[Kal60] Kalman, R. E. A new approach to linear filtering and prediction problems. J. basic Eng. 82, 35-45, 1960.

[Kim16] Kim, H., Park, J., Park, H. \& Paik, J. Fire flame detection based on color model and motion estimation. in 2016 IEEE International Conference on Consumer Electronics-Asia (ICCE-Asia) 1-2, IEEE, 2016.

[Kmu18] KMU Fire and smoke database. [Online]. Available: https://cvpr.kmu.ac.kr/dataset/dataset.htm. Accessed October 2018.

[Kou12] Koubaa, M., Elarbi, M., Ben Amar, C., Nicolas, H. Collusion, MPEG4 compression and frame dropping resistant video watermarking. Multimedia Tools and Applications, 56 (2), pp. 281-301, 2012.

[LiS17] Li, S., Liu, W., Ma, H. \& Fu, H. Multi-attribute based fire detection in diverse surveillance videos. in International Conference on Multimedia Modeling 238250, Springer, 2017.

[Mej08] Mejdoub, M., Fonteles, L., BenAmar, C., Antonini, M. Fast indexing method for image retrieval using treestructured lattices. 2008 International Workshop on Content-Based Multimedia Indexing, CBMI, Conference Proceedings, pp. 365-372, 2008.

[Mia13] Miao, L. \& Wang, A. Video flame detection algorithm based on region growing. in 2013 6th International Congress on Image and Signal Processing (CISP) 2, 1014-1018, IEEE, 2013.

[Min18] Ministry of the interior of France. Dossier de presse relatif à la campagne 2018 de lutte contre les feux de forêts. $2018 . \quad$ Available at: https://www.interieur.gouv.fr/Media/MI/Files/Actualite s/dossier-de-presse-relatif-a-la-campagne-2018-delutte-contre-les-feux-de-forets. Accessed: November 2018.

[Pel18] Peleshko, D., Maksymiv, O., Rak, T., Voloshyn, O. \& Morklyanyk, B. Core generator of hypotheses for real-time flame detecting. in 2018 IEEE Second International Conference on Data Stream Mining \& Processing (DSMP) 455-458, IEEE, 2018.

[See14] Seebamrungsat, J., Praising, S. \& Riyamongkol, P. Fire detection in the buildings using image processing. in 2014 Third ICT International Student Project Conference (ICT-ISPC) 95-98, IEEE, 2014.

[Tru12] Truong, T. X. \& Kim, J.-M. Fire flame detection in video sequences using multi-stage pattern recognition techniques. Eng. Appl. Artif. Intell. 25, 1365-1372, 2012.

[XiZ12] Xi, Z., Fang, X., Zhen, S. \& Zhibin, M. Video flame detection algorithm based On multi-feature fusion technique. in 2012 24th Chinese Control and Decision Conference (CCDC) 4291-4294, IEEE, 2012.

[Yan12] Yan, Y. Y., Gao, S. B., Wang, H. Y. \& Guo, Z. B. Contour extraction of flame for fire detection. in Advanced Materials Research 383, 1106-1110, Trans Tech Publ, 2012.

[Zho15] Zhou, Q., Yang, X. \& Bu, L. Analysis of shape features of flame and interference image in video fire detection. in 2015 Chinese Automation Congress (CAC) 633-637, IEEE, 2015. 